\title{
Review Article \\ Recent Development and Clinical Application of Cancer Vaccine: Targeting Neoantigens
}

\author{
Ren-You Pan, ${ }^{1,2}$ Wen-Hung Chung $\left(\mathbb{D},{ }^{1,2,3,4,5}\right.$ Mu-Tzu Chu, $^{6}$ Shu-Jen Chen, ${ }^{7}$ \\ Hua-Chien Chen, ${ }^{7}$ Lei Zheng $\mathbb{1},{ }^{8}$ and Shuen-Iu Hung $\mathbb{1}^{6}$ \\ ${ }^{1}$ Department of Dermatology, Drug Hypersensitivity Clinical and Research Center, Chang Gung Memorial Hospital, Linkou, \\ Taipei and Keelung, Taiwan \\ ${ }^{2}$ Chang Gung Immunology Consortium, Chang Gung Memorial Hospital and Chang Gung University, Taiwan \\ ${ }^{3}$ College of Medicine, Chang Gung University, Taoyuan, Taiwan \\ ${ }^{4}$ Whole-Genome Research Core Laboratory of Human Diseases, Chang Gung Memorial Hospital, Keelung, Taiwan \\ ${ }^{5}$ Department of Dermatology, Xiamen Chang Gung Hospital, China \\ ${ }^{6}$ Department and Institute of Pharmacology, School of Medicine, National Yang-Ming University, Taipei, Taiwan \\ ${ }^{7}$ ACT Genomics, Taipei, Taiwan \\ ${ }^{8}$ The Pancreatic Cancer Precision Medicine Center of Excellence Program, Johns Hopkins University School of Medicine, Baltimore, \\ Maryland, USA
}

Correspondence should be addressed to Shuen-Iu Hung; sihung@ym.edu.tw

Received 12 July 2018; Accepted 18 October 2018; Published 19 December 2018

Guest Editor: Lei Zhao

Copyright (C) 2018 Ren-You Pan et al. This is an open access article distributed under the Creative Commons Attribution License, which permits unrestricted use, distribution, and reproduction in any medium, provided the original work is properly cited.

Recently, increasing data show that immunotherapy could be a powerful weapon against cancers. Comparing to the traditional surgery, chemotherapy or radiotherapy, immunotherapy more specifically targets cancer cells, giving rise to the opportunities to the patients to have higher response rates and better quality of life and even to cure the disease. Cancer vaccines could be designed to target tumor-associated antigens (TAAs), cancer germline antigens, virus-associated antigens, or tumor-specific antigens (TSAs), which are also called neoantigens. The cancer vaccines could be cell-based (e.g., dendritic cell vaccine provenge (sipuleucel-T) targeting prostatic acid phosphatase for metastatic prostate cancer), peptide/protein-based, or gene- (DNA/RNA) based, with the different kinds of adjuvants. Neoantigens are tumor-specific and could be presented by MHC molecules and recognized by $\mathrm{T}$ lymphocytes, serving the ideal immune targets to increase the therapeutic specificity and decrease the risk of nonspecific autoimmunity. By targeting the shared antigens and private epitopes, the cancer vaccine has potential to treat the disease. Accordingly, personalized neoantigen-based immunotherapies are emerging. In this article, we review the literature and evidence of the advantage and application of cancer vaccine. We summarize the recent clinical trials of neoantigen cancer vaccines which were designed according to the patients' personal mutanome. With the rapid development of personalized immunotherapy, it is believed that tumors could be efficiently controlled and become curable in the new era of precision medicine.

\section{Introduction}

Cancer cells have characteristics of genetic instabilities and accumulate somatic mutations rapidly (1-4). The genome sequencing of cancer cells revealed heterogeneity, and tens to hundreds to thousands of somatic mutations amassed in individual patients. The high intertumoral heterogeneity is evidenced by The Cancer Genome Atlas (TCGA) database, which stores the genomic data of thousands of tumor specimens [1-3]. There are various types of mutations, such as point mutations, insertion/deletions, gene amplification, and translocations in cancer cells. Some of them may lead to nonsynonymous somatic mutations altering the amino acid coding sequences and creating uncontrollable and abnormal proteins to promote cell proliferation. These aberrant peptide sequences could be seen by our immune system. Tumor-specific antigens (TSAs), called as neoantigens, are created by the genomic codon alternations, editing, usage, 
antigen processing, and presentation $[4,5]$. Neoantigens could be presented by the major histocompatibility complex (MHC; also known as human leukocyte antigen (HLA) in humans) on the cell surface and recognized by the $\mathrm{T}$ lymphocytes. As neoantigens are tumor-specific and not expressed by normal cells $[4,5]$, they are ideal therapeutic targets and have great potential to maximize the therapeutic specificity, overcome the immune tolerance, and minimize the risk of autoimmunity. In this article, we review the literature of tumor antigens and cancer vaccines and also discuss the applications and values of this approach towards precision medicine.

\section{Emerging Immunotherapies for Cancer Treatments}

In recent years, immunotherapies rapidly develop and open a new era of cancer treatment. In 2011, the FDA first approved an immune checkpoint inhibitor (ICI), ipilimumab, a CTLA4 blockage, which prolonged the overall survival rate of patients with metastatic melanoma $[6,7]$. Following this line, there are increasing ICI, such as anti-PD1 and anti-PD-L1 antibodies, proven to be effective and durable therapies in subsets of patients with a variety of tumor types: metastatic melanoma, nonsmall cell lung cancer (NSCLC), prostate cancer, renal cell carcinoma, and so on [8]. The response rates of ICI, however, are correlated with the mutation load of tumors of individuals and the presence of microsatellite instability (MSI) or DNA repair enzyme deficiency [9-11]. Nevertheless, the use of ICI carries a risk to develop irAE (immune-related adverse events), which occur via nonspecific activation of the patient's immune system, leading to serious and even fatal adverse reactions $[12,13]$. More efforts are needed to improve the response rates and tumor antigen specificity of ICI and to decrease the incidence of irAE. More recently, the first chimeric antigen receptor- (CAR-) $\mathrm{T}$ cell immunotherapy, anti-CD19 CAR-T for B cell lymphoma, was approved by the FDA in Aug 2017 [14, 15]. After that, there are increasing clinical trials using CAR-T therapy to treat cancers $[16,17]$. CAR-T cells target the tumorassociated antigens (TAAs), such as CD19 on B cell malignancies [18, 19] and ERBB2 on breast cancers [20], which are also expressed on the normal cells. CAR-T therapy has the on-target but off-tumor side effect. Although CAR-T therapies have shown considerable promise in some acute lymphoid leukemia $[18,19]$, it is still a big challenge to treat solid cancers with CAR-T cells due to the lack of suitable TAAs. The reported overall objective response rates (ORR) of CAR-T therapy for solid tumors are still low [21, 22].

Targeting tumor-specific antigens (TSAs) has been considered an important therapeutic approach. As TSAs are exempt from central tolerance [23], these neoantigens could be presented by HLA and recognized by $\mathrm{T}$ lymphocytes of the immune system. Effective antitumor immunity in humans has been associated with the presence of $\mathrm{T}$ cells recognizing cancer neoantigens. The studies of adoptive cell transfer (ACT) of autologous tumor-infiltrating lymphocytes (TILs) revealed that neoantigen-specific T cells are crucial for clinical responses [24-27]. The isolated T cell clones or T cell
TABLE 1: Categories of tumor antigens.

Different antigen types, descriptions, and examples

Tumor-associated antigens (TAAs)

Low levels of expression on normal host cells

Disproportionately expressed on tumor cells

Often result from genetic amplification or posttranslational modifications

Example: CD19 on B cell malignancies

Cancer germline antigens (CGAs)/cancer testis antigens (CTA)

Absent on the normal adult cells, except in reproductive tissues such as testes, fetal ovaries, and trophoblast

Selectively expressed by various tumor types by epigenetic dysregulation

Example: NY-ESO-1 in various tumors

Virus-associated antigens

Arise in cancer cells from oncogenic viral proteins

Viral oncoproteins integrate into host cell genome, causing cell transformation and tumorigenesis

Carried by virally associated malignancies

Example: HPV E6/E7 oncoproteins

Tumor-specific antigens (TSAs)/neoantigens

Arise in cancer cells from nonsynonymous somatic mutations that result in the formation of new peptide sequences during tumorigenesis

Completely absent from normal host cells

Example: individual KRAS G12D somatic mutation

receptor- (TCR-) engineered $\mathrm{T}$ lymphocytes demonstrated the epitope patterns of neoantigens recognized by $\mathrm{T}$ cells [28-30]. There are increasing neoantigen-based cancer vaccines designed to target the unique immunogenic mutations arising in each patient's tumor [31]. Recently, two groups showed glimmers of the success of personalized cancer vaccines $[32,33]$. Both the personalized RNA mutanome vaccines and peptide-based vaccines induced poly-specific therapeutic immunity against cancer $[32,33]$. These neoantigen cancer vaccines demonstrated to be relatively safe, feasible, and capable of eliciting strong $\mathrm{T}$ cell responses to neoepitopes in patients with melanoma $[32,33]$. Treatments tailored to a person's individual cancer mutations cause the strong immune response to attack tumors.

\section{Tumor Antigens for Immunotherapy}

Regarding the targets of immunotherapy, there are different types of tumor antigens, including tumor-associated antigens (TAAs), cancer germline antigens (CGAs), virus-associated antigens, and tumor-specific antigens (TSAs) (Table 1).

Tumor-associated antigens (TAAs) are present in normal cells with low levels of expression but overexpressed on tumor cells in different patients. There are different kinds of TAAs, e.g., carcinoembryonic antigen (CEA) for GI cancer and PAP for prostate cancer. Using the universal antigens, various cancer vaccines have been designed for patients with tumor expressing the specific TAA. For example, stimuvax 
(BLP25 liposome vaccine) targeting MUC1 for NSCLC is in the phase III trial [34].

However, most attempts targeting TAAs in the cancer vaccination have met with limited success, as TAAs are normal host proteins and therefore subject to both central and peripheral tolerance mechanisms [35]. Due to the positive and negative selection, the high-affinity TCRs for TAAs are preferentially depleted, and the affinities of the remaining TCRs for TAAs are lower than that of the TCRs for foreign antigens $[36,37]$. In addition, targeting TAAs may cause autoimmune toxicities, such as colitis, severe hepatitis, renal impairment, rapid respiratory failure, and even death [38]. For example, targeting "carbonic anhydrase 9" caused severe liver toxicity, as this TAA is expressed in bile duct epithelial cells. Nevertheless, using TAAs as immunotherapy targets still has its clinical value. CAR-T therapy targeting CD19 in patients with acute lymphoblastic leukemia (ALL) showed complete remission in high proportion of patients, though life-long administration of IVIG is needed for the patients $[14,15]$.

Cancer germline antigens (CGAs), also called cancer/testis antigens (CTAs), are present in reproductive tissues, such as testes, fetal ovaries, and trophoblasts, but have limited expression on other normal tissues in adults and are generally not present on normal reproductive cells (Table 1) [35, 39]. CGAs, such as melanoma-associated antigen 3 (MAGE-A3) and NY-ESO-1 antigen, are selectively expressed by various cancers [40, 41]. However, attempts to target CGAs have met hurdles. For example, targeting MAGE-A3 resulted in severe neurological toxicity and death [42].

Some cancers have been associated with virus infection, and the viral-encoded antigens comprising the viral open reading frames are present in the tumors only but not the normal cells (Table 1). The viral oncogenes encode oncoproteins and cause cell transformation and tumorigenesis, such as Merkel cell polyomavirus- (MCPyV-) associated Merkel cell carcinoma (MCC) and human papillomavirus- (HPV-) associated cervical cancer or oropharyngeal cancer [43-45]. Targeting virus-associated antigens has been considered to be one of the effective methods for treating cancers [46-48]. Nevertheless, some virus-associated antigens showed ability to escape from the immune detection of the host $[49,50]$.

Tumor-specific antigens (TSAs; neoantigens) arise from nonsynonymous mutations and other genetic alterations in cancer cells (Table 1). Neoantigens are mutated peptides been presented by HLA on the cell surface and subsequently recognized by the immune system. TSAs are theoretically more attractive therapeutic targets because they are different from the germline and seen as nonself by the immune system. Because normal cells do not express TSAs, neoantigenspecific immune reactions are not subject to central and peripheral tolerance. In addition, targeting TSAs should be less likely to induce autoimmunity. As a result, neoantigens appear to represent the ideal targets for therapeutic cancer vaccine and $\mathrm{T}$ cell-based cancer immunotherapy. Several neoantigens have been identified from different types of cancers, including melanoma, lung cancer, hepatoma, and renal cancers $[51,52]$.

\section{Categories of Cancer Vaccines}

With the development of technologies of next-generation sequencing (NGS), it becomes apparent that human cancers are very complex, bearing thousands of mutations. By the application of platforms of immune repertoire, increasing evidence reveals that some of the tumor antigens could be recognized by the immune repertoire. Now, there are different prediction algorithms and software for the epitope mapping and $\mathrm{MHC} /$ neoantigen binding [5, 53]. Different kinds of cancer vaccines could be designed to target diverse tumor antigens, including shared antigens or private epitopes.

There are three broad types of cancer vaccines, designed in the forms of cells, proteins/peptides, and genes (Figure 1). Regarding cell-based cancer vaccines, there are (1) autologous or allogeneic whole tumor cell vaccine and (2) autologous dendritic cells (DC), pulsed or transfected with tumor antigens in different forms, such as tumor lysates, purified proteins, peptides, DNA, or RNA [54]. When using the whole tumor cells as the antigens, the cells could be inactivated by heat, chemicals, or radiation. There are different kinds of cancer vaccines using whole tumor cells, e.g., OncoVAX (Vaccinogen) for colon cancer, Reniale (LipoNova) for renal cancer, and GVAX for prostate cancer [5557]. The autologous or allogenic whole tumor cells can be genetically modified to produce immune molecules, e.g., Lucanix (belagenpumatucel-L from NovaRx) for NSCLC [58]. The phase III study of Lucanix (belagenpumatucel-L from NovaRx), however, failed to meet the endpoint in NSCLC [58]. Since the main disadvantage of whole tumor cell-cancer vaccine is nonspecificity, targeting TAA as the component of the cell-based vaccine may improve the anticancer effect. For example, the dendritic cell vaccine, provenge (sipuleucel-T), targeting PAP for metastatic castration-resistant prostate cancer, was the first FDAapproved cell-based cancer vaccine in 2010 [59]. Nevertheless, cell-based vaccines also have the limitations of the high-cost, time-consuming, and large-scale manufacturing production for individual patients $[60,61]$.

Protein/peptide-based vaccines could be composed of TAAs, CGAs, virus-associated antigens, or TSAs, with different adjuvants. The synthetic peptide vaccines are usually composed of 20-30 amino acids targeting the specific epitopes of tumor antigens. Furthermore, the tumor antigens could be modified to fuse or mix with cytokines, antibodies, or immunogenic peptides in the protein/peptide-based cancer vaccines, e.g., Oncophage for kidney cancer, melanoma, and brain cancer and Stimuvax (BLP25 liposome vaccine) targeting MUC1 for NSCLC and breast cancer [34, 62, 63]. Peptide vaccines have several advantages, such as easy synthesis with low cost, increased stability, and relative safety. Peptide vaccines have been generally demonstrated in numerous preclinical and clinical studies. However, there are obstacles of peptide vaccines needed to be overcome, which include the limitation of well-known peptide epitopes as vaccine candidates, immune evasion, weak immunogenicity of tumor antigens, and high cost for cGMP manufacturing and production of a fully personalized cancer vaccine [64-66]. 


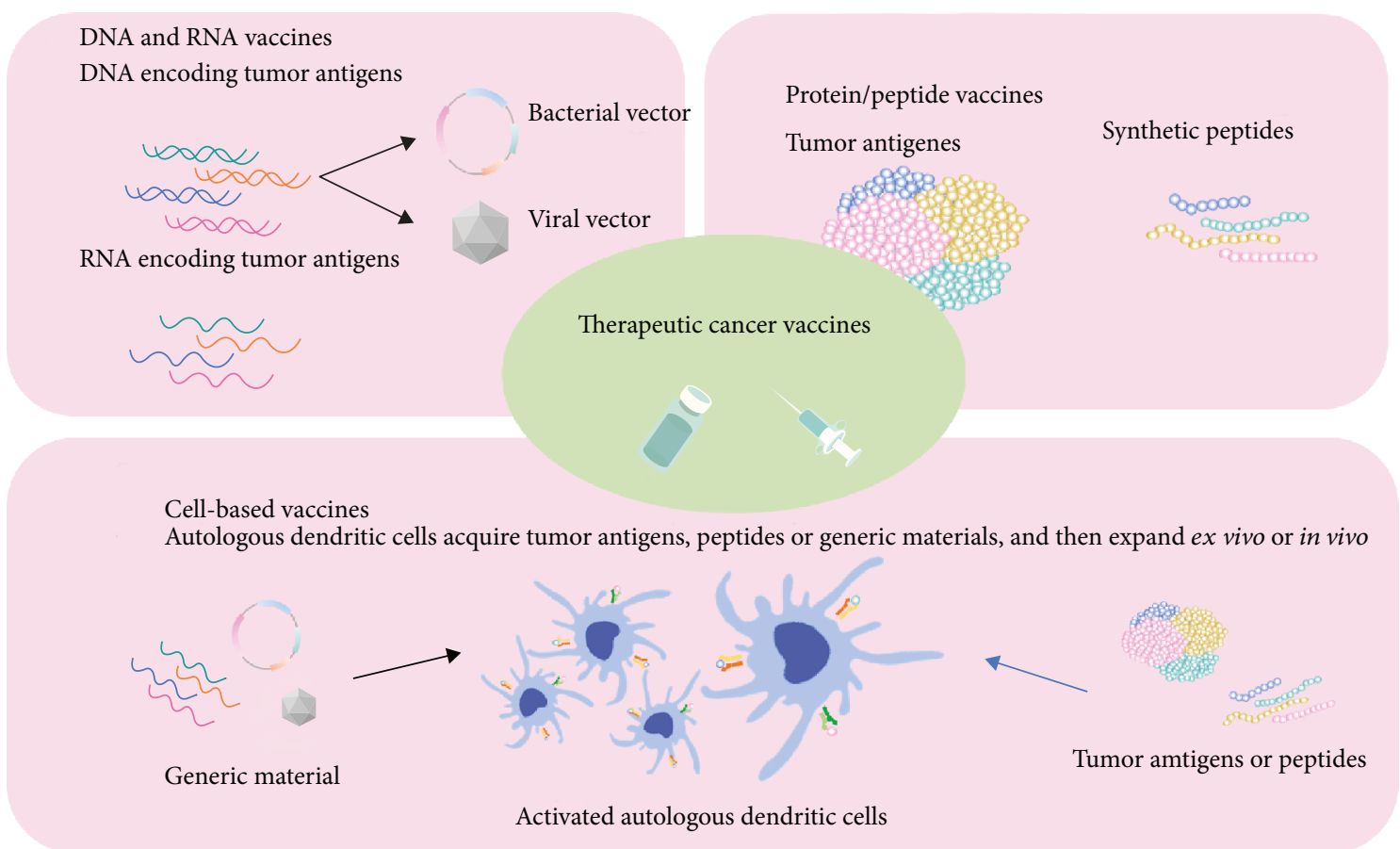

FIGURE 1: Schematic representation of different types of therapeutic cancer vaccines, which could be designed according to the forms of cells, proteins/peptides, and genes.

Gene-based cancer vaccines apply DNA (as plasmids) or RNA (as mRNA), which could be taken up by antigenpresenting cells (APC) and translated into peptides or proteins as cancer-specific antigens to stimulate the immune response. There are different kinds of DNA cancer vaccines, such as mammaglobin-A for breast cancer, PAP for prostate cancer, gp100 and gp75 DNA for melanoma, and VXM01 for pancreatic cancer $[59,67-70]$. The major obstacle of genebased vaccination is the DNA/RNA delivery method and uptake efficiency, consequently limiting the antigen transcription and presentation by APC [71]. Although electroporation or viral vectors showed higher efficiency to deliver the DNA or RNA into cells, both methods are difficult to be applied in clinical practice [72-75]. For example, the clinically approved devices for electroporation are available; however, patients' compliance has limited the use [73]. Regarding the virus-mediated delivery, it should be carefully considered for the potential side effects related to the administration of live virus together with the decreased efficiency of the presence of antiviral neutralizing antibodies in patients [72].

\section{Preclinical and Clinical Trials Applying Neoantigen-Based Cancer Vaccines}

The number of somatic mutations ranges from a few dozens to several tens of thousands in an individual tumor. With the development of NGS technologies, the highly heterogeneous neoantigens of tumor cells could be characterized. The cancer vaccine is a relatively safe and effective therapy compared to other methods of cancer treatments. To generate the personalized cancer vaccine, the somatic mutations of cancer cells could be identified by the whole exome sequencing via the comparison of the genomic DNA data of excised tumor tissue and peripheral blood mononuclear cells (PBMC) of an individual. According to the profile of detected tumor mutations, the personalized cancer vaccine could be designed to target the specific epitopes of neoantigens against cancers. The personalized cancer vaccine may consist of the synthetic peptides or genes encoding the shared tumor antigens, or private neoantigens, with the presence of adjuvants such as poly-ICLC, GM-CSF, and BCG (Figure 2). Personalized cancer may be used with the combination of other therapeutics, e.g., ICI, chemotherapy, or radiation therapy.

Based on the theory of tumor-immune cell interaction, the personalized cancer vaccination works to activate the immune system and kill cancers (Figure 2) [76]. First, the neoantigens from the cancer vaccine or died cancer cells are captured by APCs. Next, the activated APCs migrate to the lymph nodes and the MHC molecules present the neoantigens to T lymphocytes. The specific TCR recognizes the neoantigens, resulting in the priming and activation of $\mathrm{T}$ cell immunity. Neoantigen-specific $\mathrm{T}$ cells are then expanded, traffic and infiltrate to the tumor microenvironment. These expanded $\mathrm{T}$ cells specifically bind to the neoantigens of cancer cells via the interaction of the TCR/neoantigen/MHC complex. The CD4-positive $\mathrm{T}$ cells augment the immune response against cancers, and CD8-positive cytotoxic $\mathrm{T}$ lymphocytes (CTL) directly kill the cancer cells through the degranulation of granzyme, granulysin, or perforin. The lysed tumor cells release more neoantigens, which elicit the adaptive immune memory response and lead to the expansion of molecularly heterogeneous $\mathrm{T}$ cells against cancers (Figure 2). 


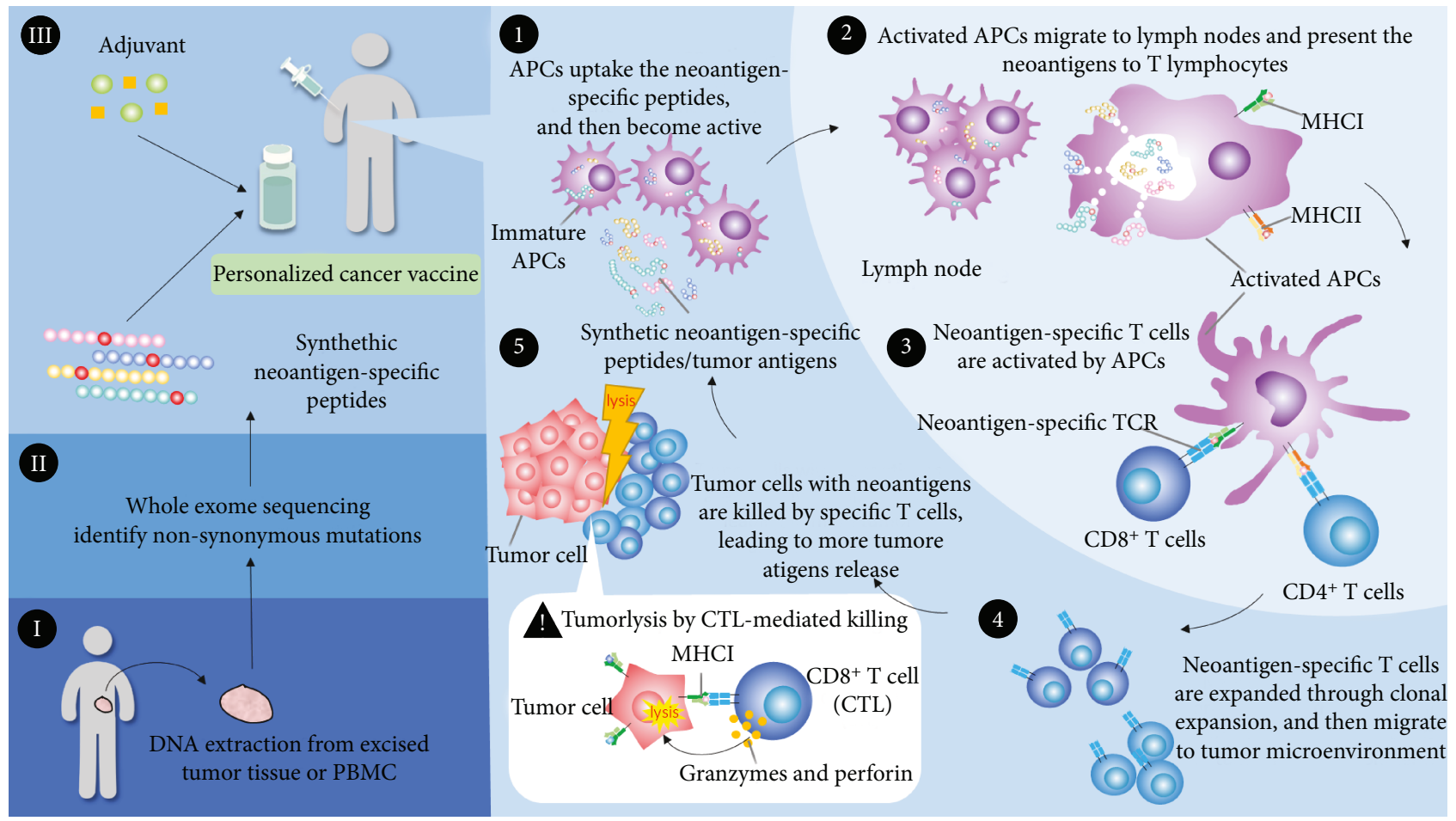

FIGURE 2: The designing strategies and immunology of personalized neoantigen cancer vaccine. (I-III) The tumor neoantigens of an individual are identified using the whole exome sequencing, and the personalized neoantigen cancer vaccine is introduced. (1) The APCs uptake the neoantigen peptides in the vaccination sites and then migrate to the lymph nodes. (2) The activated APCs present the neoantigens by MHC class I or MHC class II molecules to T cells. (3) The neoantigen-specific TCR recognizes the specific neoantigen presented by the MHC molecules of APCs. (4) The neoantigen-specific CD $4^{+}$helper or $\mathrm{CD} 8^{+}$cytotoxic T cells are activated and clonally expanded and then migrate to the tumor microenvironment. (5) The tumor cells are killed directly by neoantigen-specific $\mathrm{CD}^{+}{ }^{+}$cytotoxic $\mathrm{T}$ cells, leading to the release of more of tumor neoantigens. APC: antigen-presenting cell; MHC: major histocompatibility complex; TCR: $\mathrm{T}$ cell receptor.

In the preclinical studies of the tumor vaccination using a mouse model, Castle et al. explored the mutanome and identified candidate mutated epitopes by whole exome sequencing of the B16F10 murine melanoma (46). Fifty selected mutated gene coding peptides were vaccinated to mice, and 11 of 50 peptides demonstrated immunogenicity and induced immune responses (46). The mutated Kif18b $($ K739 N) was the dominant mutated antigen, and mice immunized with mutated Kif18b peptide showed decreased tumor progression and improved survival [77]. Yadav et al. predicted the immunogenic tumor mutations by combining mass spectrometry and exome sequencing (47). MC-38 tumor-bearing mice, which were injected with the mutated peptide vaccine (Adpgk, Reps1, and Dpagt1), showed the suppression of tumor growth [78]. Castle et al. developed a synthetic RNA pentatope vaccine (36). Each pentatope contained five 27-mer minigenes, including the mutated amino acids in the center, and each pentatope was fused to another by 10 -mer glycine-serine linker (36). The CT26 tumorbearing mice were vaccinated with the RNA pentatope, and slow disease progression and improved survival were observed (36). This study suggests that mutant MHC class II epitopes are more immunogenic and drive therapeutic immune response to cancer than that of class I epitopes.
There are clinical trials evaluating the safety and efficacy of personalized cancer vaccines. Some of the clinical trials have shown encouraging results. For example, Carreno et al. identified somatic mutations in tumors from 3 patients with melanoma by whole exome sequencing (48). The authors used an HLA binding prediction algorithm to initially filter the candidate $H L A-A^{*} 02: 01$ epitopes containing residues arising from mutations and then evaluated the MHC-epitope binding using competitive assays. The three patients received autologous dendritic cells pulsed with the top 7 neoantigen peptides, which showed higher binding affinity to the $H L A-A^{*} 02: 01$. They found that dendritic cell neoantigen vaccine increased the diversity of melanoma neoantigen-specific $\mathrm{T}$ cells (48). These neoantigens could be endogenously processed and presented to $\mathrm{T}$ cells, and the $\mathrm{T}$ lymphocytes elicited by vaccination could recognize the target cells transfected with the corresponding tandem minigene constructs [79]. Recently, Ott et al. enrolled 6 patients with melanoma and identified the tumor-specific mutations by NGS [33]. To make the personalized peptide vaccines, the authors predicted the neoantigens which could bind to the individual MHC proteins by algorithms. Each patient was vaccinated using the synthetic long peptides representing up to 20 predicted personal tumor neoantigens. The 
vaccination induced polyfunctional $\mathrm{CD} 4^{+}$and $\mathrm{CD} 8^{+} \mathrm{T}$ cells targeting $58(60 \%)$ and $15(16 \%)$ of the 97 unique neoantigens used across patients [33]. These $\mathrm{T}$ cells could discriminate mutated and wild-type antigens, and some of them could directly recognize autologous tumor [33]. Four of 6 patients had no recurrence at 25 months after vaccination, and the other two patients with recurrent disease were subsequently treated with anti-PD-1 therapy and experienced complete tumor regression [33]. In addition, Sahin et al. showed that personalized RNA mutanome vaccines elicited poly-specific therapeutic immunity against melanoma [32]. This study applied a process comprising the comprehensive identification of individual mutations, computational prediction of neoantigens with high binding affinity to MHC proteins, and designing and manufacturing of an RNA-based vaccine unique for each patient [32]. All patients developed $\mathrm{T}$ cell response against multiple vaccine neoantigens [32]. The cumulative rate of metastatic events was significantly reduced after the injection of vaccine, resulting in a sustained progression-free survival [32]. Two of 5 patients with metastatic disease had vaccine-related objective response, and a patient developed a complete response to vaccination in combination with PD-1 blockade therapy [32]. These promising results demonstrate that personalized neoantigen cancer vaccine opens a new path to cure the disease.

\section{Conclusion and Future Perspectives}

Cancer vaccine composed of unique tumor antigens specifically forces the immune system to recognize the malignancies, which could be used alone or in combination with other therapies. Among the different kinds of tumor antigens, neoantigens are ideal therapeutic targets for the design of cancer vaccine as they are tumor-specific and have the lowest risks of autoimmunity. The neoantigen-based cancer vaccines showed the induction of de novo $\mathrm{T}$ cell clones that detected multiple individual-specific neoantigens and recognized endogenously processed antigens and autologous tumor cells $[32,33]$. When the encouraging results of personalized cancer vaccines are accumulating, there are some obstacles needing to overcome. Some cancers are "cold tumors," e.g., pancreatic cancers and colorectal cancers, showing low response rates to immunotherapies. How to use personalized cancer vaccines to increase the reactive $\mathrm{T}$ cells in the microenvironment and combine with other therapies to have synergy effects on "cold tumors" needs further investigation. Another concern is the heterogeneity of tumor and immune escape. In an individual patient, the same types of neoantigens may be expressed on some, but not all tumor cells, which may cause cancers to escape from immunotherapy. One potential approach to solve this problem is to target multiple neoantigens of a diversity of malignant clones per patient, as demonstrated in the previous studies $[32,33]$. Therefore, all tumor cells could be destroyed at the same time of the treatment course, and the cancer vaccine minimizes the chance of tumor escape by the loss of antigens $[32,33]$. Lastly, the pharmacoeconomics is also an important issue for implementing personalized neoantigen cancer vaccine into clinic practice. The individualized vaccine is still expensive due to the cost for genome sequencing and manufacturing of small and personalized-specific GMP drug product batches. However, the expense for personalized cancer vaccine may reduce following the development of improved methods for predicting antigen presentation, the process of commercialization, full automation, and optimization of manufacturing processes. After having more understandings of the cancer immunology, the cancer vaccine may be designed to target the driver mutations or shared antigens of different tumor types or individuals, to increase the therapeutic efficiency, and to reduce the expense of manufacturing [80]. In conclusion, there are increasing evidence demonstrating the feasibility, safety, and immunogenicity of the personalized cancer vaccine in the treatment of cancer patients. The personalized cancer vaccine could work alone or in combination with other therapies to enhance the strength and persistence of antitumor effects, increase the survival rates and quality of life, and ultimately improve the efficacy of cancer treatments in the patients. It is anticipated that personalized cancer vaccine will make precision medicine to be available and affordable for most of patient population in the near future.

$\begin{array}{ll}\text { Abbreviations } \\ \text { ACT: } & \text { Adoptive cell transfer } \\ \text { APC: } & \text { Antigen-presenting cells } \\ \text { CAR-T therapy: } & \text { Chimeric antigen receptors-T cell therapy } \\ \text { CGAs: } & \text { Cancer germline antigens } \\ \text { CTL: } & \text { Cytotoxic T lymphocyte } \\ \text { HLA: } & \text { Human leukocyte antigen } \\ \text { ICI: } & \text { Immune checkpoint inhibitors } \\ \text { irAE: } & \text { Immune-related adverse events } \\ \text { MHC: } & \text { Major histocompatibility complex } \\ \text { NSCLC: } & \text { Nonsmall cell lung cancer } \\ \text { NGS: } & \text { Next-generation sequencing } \\ \text { TAA: } & \text { Tumor-associated antigen } \\ \text { TCR: } & \text { T cell receptor } \\ \text { TIL: } & \text { Tumor-infiltrating lymphocytes } \\ \text { TSA: } & \text { Tumor-specific antigen. }\end{array}$

\section{Conflicts of Interest}

All authors declare that no conflict of interest exists.

\section{Authors' Contributions}

Ren-You Pan and Shuen-Iu Hung are responsible for the conception and design. Shuen-Iu Hung and Wen-Hung Chung are responsible for the administrative support. All authors provided the study materials or patients; collected, assembled, analyzed, and interpreted the data; and approved the final manuscript. Ren-You Pan and Wen-Hung Chung contribute equally to this work.

\section{Acknowledgments}

The authors would like to thank the National Science Council of Taiwan (MOST104-2314-B-182A-148-MY3, MOST104- 
2325-B-182A-006, MOST104-2320-B-010-036-MY3, and MOST105-2628-B-010-007-MY3) for kindly supporting this work.

\section{References}

[1] R. Akbani, K. C. Akdemir, B. A. Aksoy et al., "Genomic classification of cutaneous melanoma," Cell, vol. 161, no. 7, pp. 1681-1696, 2015.

[2] M. S. Lawrence, P. Stojanov, C. H. Mermel et al., "Discovery and saturation analysis of cancer genes across 21 tumour types," Nature, vol. 505, no. 7484, pp. 495-501, 2014.

[3] M. S. Lawrence, P. Stojanov, P. Polak et al., "Mutational heterogeneity in cancer and the search for new cancerassociated genes," Nature, vol. 499, no. 7457, pp. 214-218, 2013.

[4] L. D. Wood, D. W. Parsons, S. Jones et al., "The genomic landscapes of human breast and colorectal cancers," Science, vol. 318, no. 5853, pp. 1108-1113, 2007.

[5] P. Bais, S. Namburi, D. M. Gatti, X. Zhang, and J. H. Chuang, "CloudNeo: a cloud pipeline for identifying patient-specific tumor neoantigens," Bioinformatics, vol. 33, no. 19, pp. 3110-3112, 2017.

[6] F. S. Hodi, S. J. O'Day, D. F. McDermott et al., "Improved survival with ipilimumab in patients with metastatic melanoma," The New England Journal of Medicine, vol. 363, no. 8, pp. 711-723, 2010.

[7] C. Robert, L. Thomas, I. Bondarenko et al., "Ipilimumab plus dacarbazine for previously untreated metastatic melanoma," The New England Journal of Medicine, vol. 364, no. 26, pp. 2517-2526, 2011.

[8] S. H. Baumeister, G. J. Freeman, G. Dranoff, and A. H. Sharpe, "Coinhibitory pathways in immunotherapy for cancer," Annual Review of Immunology, vol. 34, no. 1, pp. 539-573, 2016.

[9] M. Yarchoan, A. Hopkins, and E. M. Jaffee, "Tumor mutational burden and response rate to PD-1 inhibition," The New England Journal of Medicine, vol. 377, no. 25, pp. 25002501, 2017.

[10] S. Vranic, "Microsatellite instability status predicts response to anti-PD-1/PD-L1 therapy regardless the histotype: a comment on recent advances," Bosnian Journal of Basic Medical Sciences, vol. 17, no. 3, pp. 274-275, 2017.

[11] V. Lee, A. Murphy, D. T. le, and L. A. Diaz Jr, "Mismatch repair deficiency and response to immune checkpoint blockade," The Oncologist, vol. 21, no. 10, pp. 1200-1211, 2016.

[12] D. M. Pardoll, "The blockade of immune checkpoints in cancer immunotherapy," Nature Reviews. Cancer, vol. 12, no. 4, pp. 252-264, 2012.

[13] L. A. Fecher, S. S. Agarwala, F. S. Hodi, and J. S. Weber, "Ipilimumab and its toxicities: a multidisciplinary approach," The Oncologist, vol. 18, no. 6, pp. 733-743, 2013.

[14] M. B. Geyer, "First CAR to Pass the Road Test: Tisagenlecleucel's Drive to FDA Approval," Clinical Cancer Research, 2018.

[15] A. Mullard, "FDA approves first CAR T therapy," Nature Reviews Drug Discovery, vol. 16, no. 10, p. 669, 2017.

[16] D. Pettitt, Z. Arshad, J. Smith, T. Stanic, G. Hollander, and D. Brindley, "CAR-T cells: a systematic review and mixed methods analysis of the clinical trial landscape," Molecular Therapy, vol. 26, no. 2, pp. 342-353, 2018.
[17] J. Hartmann, M. Schussler-Lenz, A. Bondanza, and C. J. Buchholz, "Clinical development of CAR T cells-challenges and opportunities in translating innovative treatment concepts," EMBO Molecular Medicine, vol. 9, no. 9, pp. 1183-1197, 2017.

[18] S. A. Grupp, M. Kalos, D. Barrett et al., "Chimeric antigen receptor-modified $\mathrm{T}$ cells for acute lymphoid leukemia," The New England Journal of Medicine, vol. 368, no. 16, pp. 15091518, 2013.

[19] S. L. Maude, N. Frey, P. A. Shaw et al., "Chimeric antigen receptor $\mathrm{T}$ cells for sustained remissions in leukemia," The New England Journal of Medicine, vol. 371, no. 16, pp. 15071517, 2014.

[20] D. J. Slamon, W. Godolphin, L. A. Jones et al., "Studies of the HER-2/neu proto-oncogene in human breast and ovarian cancer," Science, vol. 244, no. 4905, pp. 707-712, 1989.

[21] K. Newick, E. Moon, and S. M. Albelda, "Chimeric antigen receptor T-cell therapy for solid tumors," Molecular Therapy - Oncolytics, vol. 3, article 16006, 2016.

[22] A. L. Xia, X. C. Wang, Y. J. Lu, X. J. Lu, and B. Sun, "Chimericantigen receptor $\mathrm{T}$ (CAR-T) cell therapy for solid tumors: challenges and opportunities," Oncotarget, vol. 8, no. 52, pp. 90521-90531, 2017.

[23] L. Klein, B. Kyewski, P. M. Allen, and K. A. Hogquist, "Positive and negative selection of the T cell repertoire: what thymocytes see (and don't see)," Nature Reviews. Immunology, vol. 14, no. 6, pp. 377-391, 2014.

[24] E. Tran, S. Turcotte, A. Gros et al., "Cancer immunotherapy based on mutation-specific CD4+ T cells in a patient with epithelial cancer," Science, vol. 344, no. 6184, pp. 641-645, 2014.

[25] N. van Rooij, M. M. van Buuren, D. Philips et al., "Tumor exome analysis reveals neoantigen-specific T-cell reactivity in an ipilimumab-responsive melanoma," Journal of Clinical Oncology, vol. 31, no. 32, pp. e439-e442, 2013.

[26] M. M. Gubin, M. N. Artyomov, E. R. Mardis, and R. D. Schreiber, "Tumor neoantigens: building a framework for personalized cancer immunotherapy," The Journal of Clinical Investigation, vol. 125, no. 9, pp. 3413-3421, 2015.

[27] T. N. Schumacher and R. D. Schreiber, "Neoantigens in cancer immunotherapy," Science, vol. 348, no. 6230, pp. 69-74, 2015.

[28] T. Blankenstein, M. Leisegang, W. Uckert, and H. Schreiber, "Targeting cancer-specific mutations by $\mathrm{T}$ cell receptor gene therapy," Current Opinion in Immunology, vol. 33, pp. 112119,2015

[29] E. Stronen, M. Toebes, S. Kelderman et al., "Targeting of cancer neoantigens with donor-derived $\mathrm{T}$ cell receptor repertoires," Science, vol. 352, no. 6291, pp. 1337-1341, 2016.

[30] M. T. Bethune and A. V. Joglekar, "Personalized T cellmediated cancer immunotherapy: progress and challenges," Current Opinion in Biotechnology, vol. 48, pp. 142-152, 2017.

[31] E. F. Fritsch, N. Hacohen, and C. J. Wu, "Personal neoantigen cancer vaccines: the momentum builds," OncoImmunology, vol. 3, no. 6, article e29311, 2014.

[32] U. Sahin, E. Derhovanessian, M. Miller et al., "Personalized RNA mutanome vaccines mobilize poly-specific therapeutic immunity against cancer," Nature, vol. 547, no. 7662, pp. 222-226, 2017.

[33] P. A. Ott, Z. Hu, D. B. Keskin et al., "An immunogenic personal neoantigen vaccine for patients with melanoma," Nature, vol. 547, no. 7662, pp. 217-221, 2017.

[34] C. Butts, M. A. Socinski, P. L. Mitchell et al., "Tecemotide (LBLP25) versus placebo after chemoradiotherapy for stage III 
non-small-cell lung cancer (START): a randomised, doubleblind, phase 3 trial," The Lancet Oncology, vol. 15, no. 1, pp. 59-68, 2014.

[35] P. G. Coulie, B. J. Van den Eynde, P. van der Bruggen, and T. Boon, "Tumour antigens recognized by $\mathrm{T}$ lymphocytes: at the core of cancer immunotherapy," Nature Reviews. Cancer, vol. 14, no. 2, pp. 135-146, 2014.

[36] J. D. Stone, D. T. Harris, and D. M. Kranz, "TCR affinity for p/ MHC formed by tumor antigens that are self-proteins: impact on efficacy and toxicity," Current Opinion in Immunology, vol. 33, pp. 16-22, 2015.

[37] M. Aleksic, N. Liddy, P. E. Molloy et al., "Different affinity windows for virus and cancer-specific T-cell receptors: implications for therapeutic strategies," European Journal of Immunology, vol. 42, no. 12, pp. 3174-3179, 2012.

[38] J. P. Ward, M. M. Gubin, and R. D. Schreiber, "The role of neoantigens in naturally occurring and therapeutically induced immune responses to cancer," Advances in Immunology, vol. 130, pp. 25-74, 2016.

[39] A. J. G. Simpson, O. L. Caballero, A. Jungbluth, Y. T. Chen, and L. J. Old, "Cancer/testis antigens, gametogenesis and cancer," Nature Reviews. Cancer, vol. 5, no. 8, pp. 615625, 2005.

[40] P. Chomez, O. De Backer, M. Bertrand, E. De Plaen, T. Boon, and S. Lucas, "An overview of the MAGE gene family with the identification of all human members of the family," Cancer Research, vol. 61, no. 14, pp. 5544-5551, 2001.

[41] S. Gnjatic, H. Nishikawa, A. A. Jungbluth et al., "NY-ESO-1: review of an immunogenic tumor antigen," Advances in Cancer Research, vol. 95, pp. 1-30, 2006.

[42] R. A. Morgan, N. Chinnasamy, D. Abate-Daga et al., "Cancer regression and neurological toxicity following anti-MAGEA3 TCR gene therapy," Journal of Immunotherapy, vol. 36, no. 2, pp. 133-151, 2013.

[43] H. Feng, M. Shuda, Y. Chang, and P. S. Moore, "Clonal integration of a polyomavirus in human Merkel cell carcinoma," Science, vol. 319, no. 5866, pp. 1096-1100, 2008.

[44] M. L. Gillison, W. M. Koch, R. B. Capone et al., "Evidence for a causal association between human papillomavirus and a subset of head and neck cancers," Journal of the National Cancer Institute, vol. 92, no. 9, pp. 709-720, 2000.

[45] J. M. M. Walboomers, M. V. Jacobs, M. M. Manos et al., "Human papillomavirus is a necessary cause of invasive cervical cancer worldwide," The Journal of Pathology, vol. 189, no. 1, pp. 12-19, 1999.

[46] X. G. Wang, E. Revskaya, R. A. Bryan et al., "Treating cancer as an infectious disease-viral antigens as novel targets for treatment and potential prevention of tumors of viral etiology," PLoS One, vol. 2, no. 10, article e1114, 2007.

[47] M. E. McLaughlin-Drubin and K. Munger, "Viruses associated with human cancer," Biochimica et Biophysica Acta (BBA) - Molecular Basis of Disease, vol. 1782, no. 3, pp. 127-150, 2008.

[48] M. Kuroki and N. Shirasu, "Novel treatment strategies for cancer and their tumor-targeting approaches using antibodies against tumor-associated antigens," Anticancer Research, vol. 34, no. 8, pp. 4481-4488, 2014.

[49] M. Lucas, U. Karrer, A. Lucas, and P. Klenerman, "Viral escape mechanisms-escapology taught by viruses," International Journal of Experimental Pathology, vol. 82, no. 5, pp. 269286, 2001.
[50] B. B. Finlay and G. McFadden, "Anti-immunology: evasion of the host immune system by bacterial and viral pathogens," Cell, vol. 124, no. 4, pp. 767-782, 2006.

[51] M. Takenoyama, J. F. Baurain, M. Yasuda et al., "A point mutation in the NFYC gene generates an antigenic peptide recognized by autologous cytolytic $\mathrm{T}$ lymphocytes on a human squamous cell lung carcinoma," International Journal of Cancer, vol. 118, no. 8, pp. 1992-1997, 2006.

[52] X. Zhou, D. Y. Jun, A. M. Thomas et al., "Diverse CD8 ${ }^{+}$T-cell responses to renal cell carcinoma antigens in patients treated with an autologous granulocyte-macrophage colonystimulating factor gene-transduced renal tumor cell vaccine," Cancer Research, vol. 65, no. 3, pp. 1079-1088, 2005.

[53] E. Tappeiner, F. Finotello, P. Charoentong, C. Mayer, D. Rieder, and Z. Trajanoski, "TIminer: NGS data mining pipeline for cancer immunology and immunotherapy," Bioinformatics, vol. 33, no. 19, pp. 3140-3141, 2017.

[54] L. Galluzzi, E. Vacchelli, J. M. B. S. Pedro et al., "Classification of current anticancer immunotherapies," Oncotarget, vol. 5, no. 24, pp. 12472-12508, 2014.

[55] M. G. Hanna Jr., "Immunotherapy with autologous tumor cell vaccines for treatment of occult disease in early stage colon cancer," Human Vaccines \& Immunotherapeutics, vol. 8, no. 8, pp. 1156-1160, 2012.

[56] S. Wittke, S. Baxmann, D. Fahlenkamp, and S. T. Kiessig, "Tumor heterogeneity as a rationale for a multi-epitope approach in an autologous renal cell cancer tumor vaccine," OncoTargets and Therapy, vol. 9, pp. 523-537, 2016.

[57] S. M. Geary and A. K. Salem, "Prostate cancer vaccines: update on clinical development," OncoImmunology, vol. 2, no. 5, article e24523, 2014.

[58] C. Zappa and S. A. Mousa, "Non-small cell lung cancer: current treatment and future advances," Translational Lung Cancer Research, vol. 5, no. 3, pp. 288-300, 2016.

[59] M. A. Cheever and C. S. Higano, "PROVENGE (Sipuleucel-T) in prostate cancer: the first FDA-approved therapeutic cancer vaccine," Clinical Cancer Research, vol. 17, no. 11, pp. 3520 3526, 2011.

[60] P. L. Lollini, F. Cavallo, P. Nanni, and E. Quaglino, "The promise of preventive cancer vaccines," Vaccines, vol. 3, no. 2, pp. 467-489, 2015.

[61] D. W. Mullins, S. L. Sheasley, R. M. Ream, T. N. J. Bullock, Y.-X. Fu, and V. H. Engelhard, "Route of immunization with peptide-pulsed dendritic cells controls the distribution of memory and effector $\mathrm{T}$ cells in lymphoid tissues and determines the pattern of regional tumor control," The Journal of Experimental Medicine, vol. 198, no. 7, pp. 10231034, 2003.

[62] A. di Pietro, G. Tosti, P. F. Ferrucci, and A. Testori, "Oncophage: step to the future for vaccine therapy in melanoma," Expert Opinion on Biological Therapy, vol. 8, no. 12, pp. 1973-1984, 2008.

[63] W. Xia, J. Wang, Y. Xu, F. Jiang, and L. Xu, "L-BLP25 as a peptide vaccine therapy in non-small cell lung cancer: a review," Journal of Thoracic Disease, vol. 6, no. 10, pp. 1513-1520, 2014.

[64] M. Larche, "Peptide immunotherapy for allergic diseases," Allergy, vol. 62, no. 3, pp. 325-331, 2007.

[65] S. Mocellin, P. Pilati, and D. Nitti, "Peptide-based anticancer vaccines: recent advances and future perspectives," Current Medicinal Chemistry, vol. 16, no. 36, pp. 4779-4796, 2009. 
[66] H. Yang and D. S. Kim, "Peptide immunotherapy in vaccine development: from epitope to adjuvant," Advances in Protein Chemistry and Structural Biology, vol. 99, pp. 1-14, 2015.

[67] T. P. Fleming and M. A. Watson, "Mammaglobin, a breastspecific gene, and its utility as a marker for breast cancer," Annals of the New York Academy of Sciences, vol. 923, pp. 78-89, 2000.

[68] G. Ghanem and J. Fabrice, "Tyrosinase related protein 1 (TYRP1/gp75) in human cutaneous melanoma," Molecular Oncology, vol. 5, no. 2, pp. 150-155, 2011.

[69] D. R. Minor, "gp100 peptide vaccine in melanoma," The New England Journal of Medicine, vol. 365, no. 8, p. 771, 2011.

[70] F. H. Schmitz-Winnenthal, N. Hohmann, A. G. Niethammer et al., "Anti-angiogenic activity of VXM01, an oral T-cell vaccine against VEGF receptor 2, in patients with advanced pancreatic cancer: a randomized, placebo-controlled, phase 1 trial," OncoImmunology, vol. 4, no. 4, article e1001217, 2015.

[71] L. Aurisicchio and G. Ciliberto, "Genetic cancer vaccines: current status and perspectives," Expert Opinion on Biological Therapy, vol. 12, no. 8, pp. 1043-1058, 2012.

[72] T. Osada, M. A. Morse, A. Hobeika, and H. K. Lyerly, "Novel recombinant alphaviral and adenoviral vectors for cancer immunotherapy," Seminars in Oncology, vol. 39, no. 3, pp. 305-310, 2012.

[73] S. H. Lee, S. N. Danishmalik, and J. I. Sin, "DNA vaccines, electroporation and their applications in cancer treatment," Human Vaccines \& Immunotherapeutics, vol. 11, no. 8, pp. 1889-1900, 2015.

[74] C. Curcio, A. S. Khan, A. Amici et al., "DNA immunization using constant-current electroporation affords long-term protection from autochthonous mammary carcinomas in cancerprone transgenic mice," Cancer Gene Therapy, vol. 15, no. 2, pp. 108-114, 2008.

[75] S. Rolla, C. Marchini, S. Malinarich et al., "Protective immunity against neu-positive carcinomas elicited by electroporation of plasmids encoding decreasing fragments of rat neu extracellular domain," Human Gene Therapy, vol. 19, no. 3, pp. 229-240, 2008.

[76] D. S. Chen and I. Mellman, "Oncology meets immunology: the cancer-immunity cycle," Immunity, vol. 39, no. 1, pp. 1-10, 2013.

[77] J. C. Castle, S. Kreiter, J. Diekmann et al., "Exploiting the mutanome for tumor vaccination," Cancer Research, vol. 72, no. 5, pp. 1081-1091, 2012.

[78] M. Yadav, S. Jhunjhunwala, Q. T. Phung et al., "Predicting immunogenic tumour mutations by combining mass spectrometry and exome sequencing," Nature, vol. 515, no. 7528, pp. 572-576, 2014.

[79] B. M. Carreno, V. Magrini, M. Becker-Hapak et al., "Cancer immunotherapy. A dendritic cell vaccine increases the breadth and diversity of melanoma neoantigen-specific T cells," Science, vol. 348, no. 6236, pp. 803-808, 2015.

[80] Y. C. Lu, X. Yao, J. S. Crystal et al., "Efficient identification of mutated cancer antigens recognized by $\mathrm{T}$ cells associated with durable tumor regressions," Clinical Cancer Research, vol. 20, no. 13, pp. 3401-3410, 2014. 


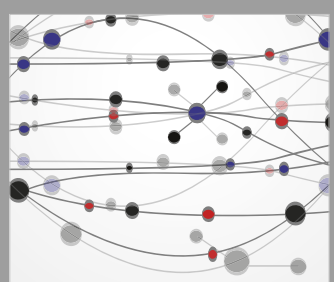

The Scientific World Journal
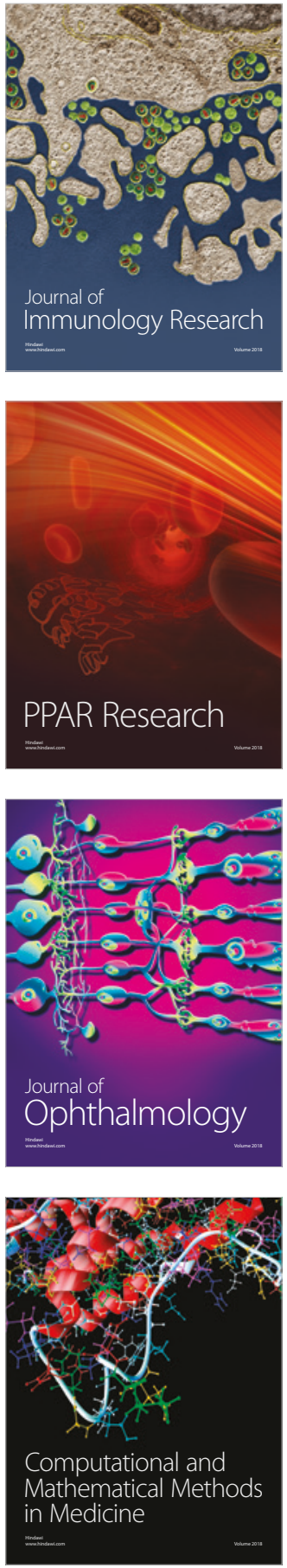

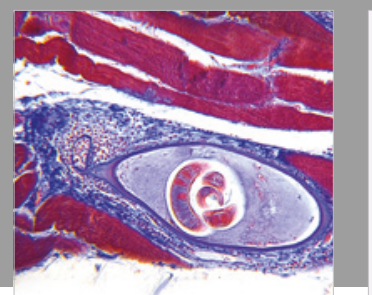

Gastroenterology Research and Practice

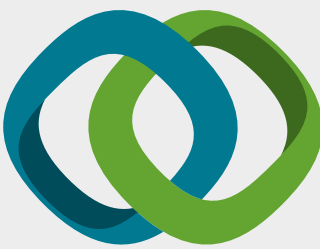

\section{Hindawi}

Submit your manuscripts at

www.hindawi.com
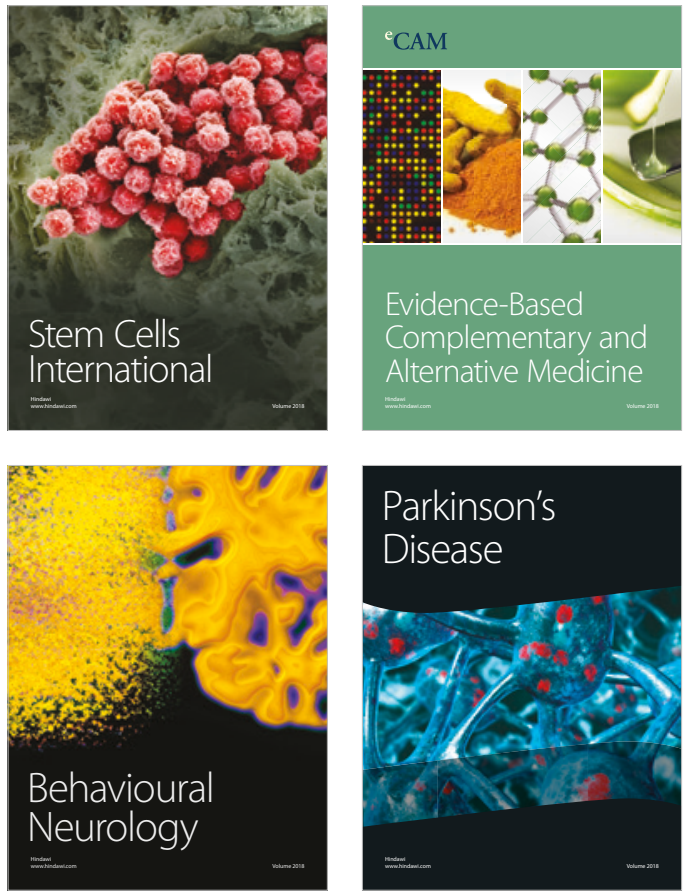

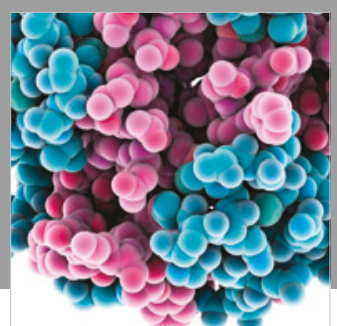

ournal of

Diabetes Research

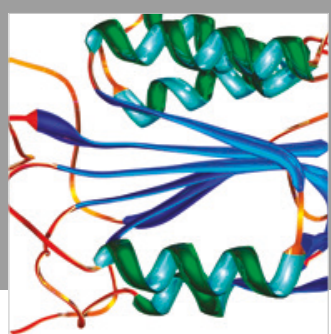

Disease Markers
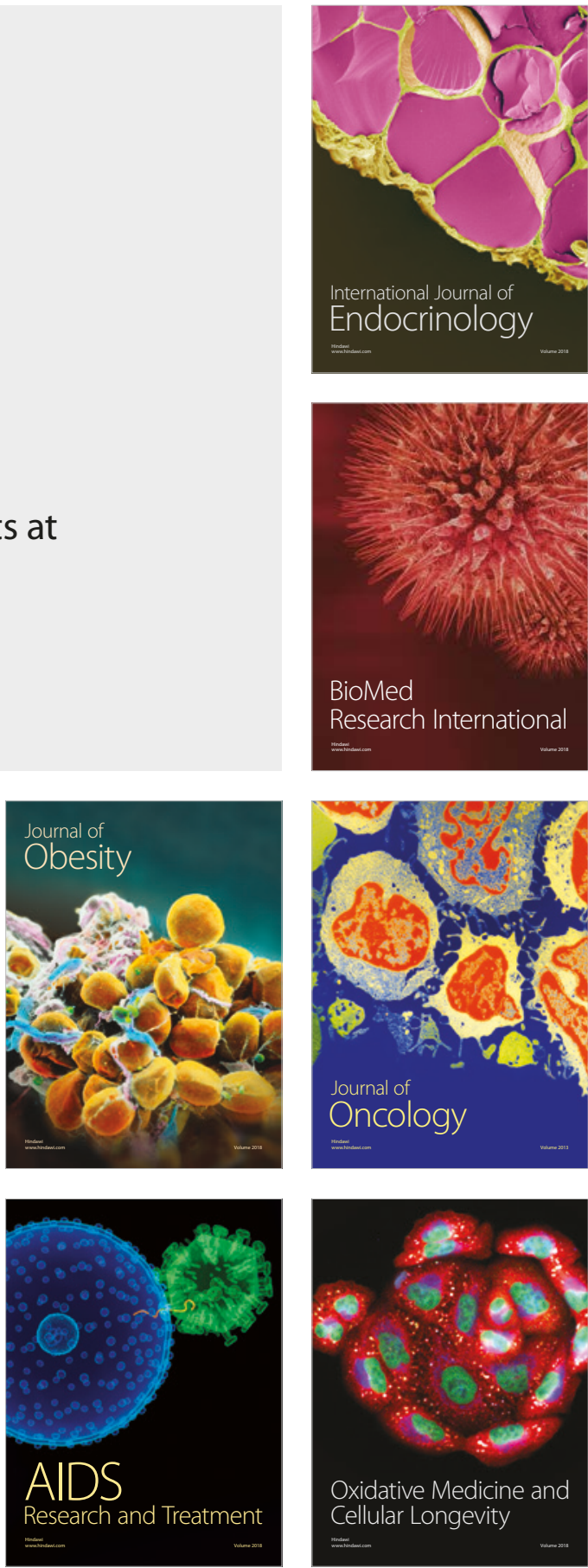